\title{
Community Health Workers and Medicaid Managed Care in New Mexico
}

\author{
Diane Johnson - Patricia Saavedra $\cdot$ Eugene Sun - Ann Stageman • \\ Dodie Grovet $\cdot$ Charles Alfero $\cdot$ Carmen Maynes $\cdot$ Betty Skipper · \\ Wayne Powell $\cdot$ Arthur Kaufman
}

Published online: 28 September 2011

(C) The Author(s) 2011. This article is published with open access at Springerlink.com

\begin{abstract}
We describe the impact of community health workers (CHWs) providing community-based support services to enrollees who are high consumers of health resources in a Medicaid managed care system. We conducted a retrospective study on a sample of 448 enrollees who were assigned to field-based CHWs in 11 of New Mexico's 33 counties. The CHWs provided patients education, advocacy and social support for a period up to 6 months. Data was collected on services provided, and community resources accessed. Utilization and payments in the emergency department, inpatient service, non-narcotic and narcotic prescriptions as well as outpatient primary care and specialty care were collected on each patient for a 6 month period before, for 6 months during and for 6 months after the intervention. For comparison, data was collected on another group of 448 enrollees who were also high consumers of health resources but who did not receive CHW intervention. For all measures, there was a significant reduction in both numbers of claims and payments after the community health worker intervention. Costs also declined in the non-CHW group on all measures, but to a more modest degree, with a greater reduction than in the CHW group in use of ambulatory services. The incorporation of field-based, community health workers as part of
\end{abstract}

\footnotetext{
D. Johnson · P. Saavedra · B. Skipper · W. Powell ·

A. Kaufman ( $\square)$

University of New Mexico, Room 137 MSC 09 5065,

Albuquerque, NM 87131, USA

e-mail: akaufman@salud.unm.edu

E. Sun · A. Stageman - D. Grovet

Molina Healthcare of New Mexico, Albuquerque, NM, USA

C. Alfero - C. Maynes

Hidalgo Medical Services, Hidalgo, NM, USA
}

Medicaid managed care to provide supportive services to high resource-consuming enrollees can improve access to preventive and social services and may reduce resource utilization and cost.

Keywords Community health workers - Managed care

\section{Introduction}

Community Health Workers (CHWs) are lay members of communities who serve for pay or as volunteers in association with the local health care system in both urban and rural environments and usually share ethnicity, language, socioeconomic status, and life experiences with the community members served. CHWs are identified by many titles such as community health advisors, lay health advocates, "promotores(as)," patient navigators, community health representatives, peer health promoters, and peer health educators. CHWs deliver a diversity of services through education, advocacy, and social support. These services include increasing access to preventive care by connecting members to a medical home, teaching concepts of prevention and chronic disease management, encouraging members to make healthier lifestyle choices, helping members keep their medical appointments, and increasing adherence to treatment regimens [1]. They can serve as a bridge between hospitals or clinics and communities and they are forming a growing part of the US healthcare workforce [2, 3].

The roles of CHWs vary considerably. Some serve voluntarily and some are supported by grant-funding from a variety of sources. Others may focus on needs of specific subsets of the population such as maternal and infant care [4] or on specific health risks such as smoking [5] or on a 
single chronic disease such as hypertension, diabetes or depression [6]. Interest in incorporating CHWs into the health workforce is growing as a strategy for improving access while decreasing cost, especially in an environment of primary care scarcity [2]. Recognition of the importance of CHWs in the healthcare workforce was enhanced by the recent designation of CHWs by the US Department of Labor [7] and by the incorporation into the Patient Protection and Affordability Care Act of a national demonstration grant for inclusion of CHWs into practices [8].

Despite the services and value that CHWs provide, reimbursement for CHWs is often tenuous and inconsistent. This is a consequence of their work often being funded by grants which are by nature limited, and by the fact that in most states, CHWs are not allowed to bill Medicaid or Medicare directly for their services. The desirability of a CHW career is thus diminished due to financial insecurity. Moreover, the appeal of CHW services to payers of healthcare has heretofore been nominal due to the lack of conclusive studies demonstrating their effectiveness in improving the quality and cost of healthcare for underserved populations [9]. By institutionalizing CHW roles within managed care organizations, two objectives may be met. The first is to stabilize the financial compensation for members of this emerging workforce. The second relates to access to utilization data, necessary in assessing whether the work of CHWs leads to improved quality of care at lower costs.

Molina Healthcare, Inc. is a family owned, publicly traded, managed care organization with corporate headquarters in California. It is the largest family participating Hispanic-owned business in the United States. The company operates in 10 states and focuses solely on government sponsored health programs including Medicaid and Medicare. Molina Healthcare of New Mexico (MHNM) is one of seven New Mexico Medicaid Managed Care provider organizations and has taken the lead in exploring the role of CHWs within contracted provider networks.

Community Access to Resources and Education in New Mexico (CARE NM) is a non-profit organization which has operated for 8 years. It is a consortium of primary care, physical and behavioral health, public health and social service agencies. Its goal is to create a seamless system of care and universal access for the region's uninsured and underserved population. The University of New Mexico Health Sciences Center (UNM HSC) is a key member of CARE NM.

From its beginning, CARE NM has sought to develop innovative ways to meet the health care needs of the most vulnerable members of the community. Originally funded by federal Health and Human Resources Administration grants and support from the WK Kellogg Foundation's Community Voices-NM initiative, the organization has generated a number of programs improving access to basic health services including the Primary Care Dispatch, a web-based tool which locates medical homes for uninsured patients [10] and NurseAdvice New Mexico-a unique, statewide 24/7 nurse-run health advice line, supported by a public-private partnership which provides nurse advice free to any caller $[11,12]$.

In 2004, UNM HSC Department of Family and Community Medicine (DFCM), with its extensive community contacts through CARE NM, was approached by MHNM to help them improve the healthcare for members who were not seeking care through their primary care provider, but rather were repeatedly seeking care for non urgent, non emergent conditions in hospital emergency departments (EDs).

MHNM was aware of the goals and objectives of CARE NM and UNM HSC DFCM, and believed that the CHW program would be of value in contacting and helping members to understand how to navigate the health care system and access their primary care provider (PCP) for primary health care needs. The immediate objective was to decrease Emergency Department visits for non emergent conditions and to ensure appropriate management of chronic diseases such as diabetes with the primary goal of improving quality of life.

This paper reports on how UNM HSC DFCM built upon the request to train CHWs as case finders into a system of more broadly trained health workers and a more effective model of "Client Support Assistants" in collaboration with MHNM. It describes the intervention model employed in urban and rural areas of the state and preliminary outcomes regarding resources utilization, cost and savings.

\section{Methods}

MHNM negotiated with the state Medical Assistance Division to establish a billing code for the program to reimburse CHWs. UNM HSC DFCM and MHNM negotiated a standard 2-year renewable contract under which UNM would invoice MHNM for the services of their CHWs, now called "Client Support Assistants." The Assistants provide a specified set of services to the member population listed in Table 1.

A contract began in May, 2005 with an initial capitated payment structure of $\$ 256$ per member per month of service, raised to $\$ 306$ in 2007 and $\$ 321$ in 2009 . The duration of service ranged from 1 to 6 months, depending on member needs.

MHNM identified members who were high users of such health services as the ED, who had high consumption of controlled substances, who had poorly controlled chronic diseases such as diabetes, cardiovascular disease and 
Table 1 Client support assistant services through education, advocacy and social support

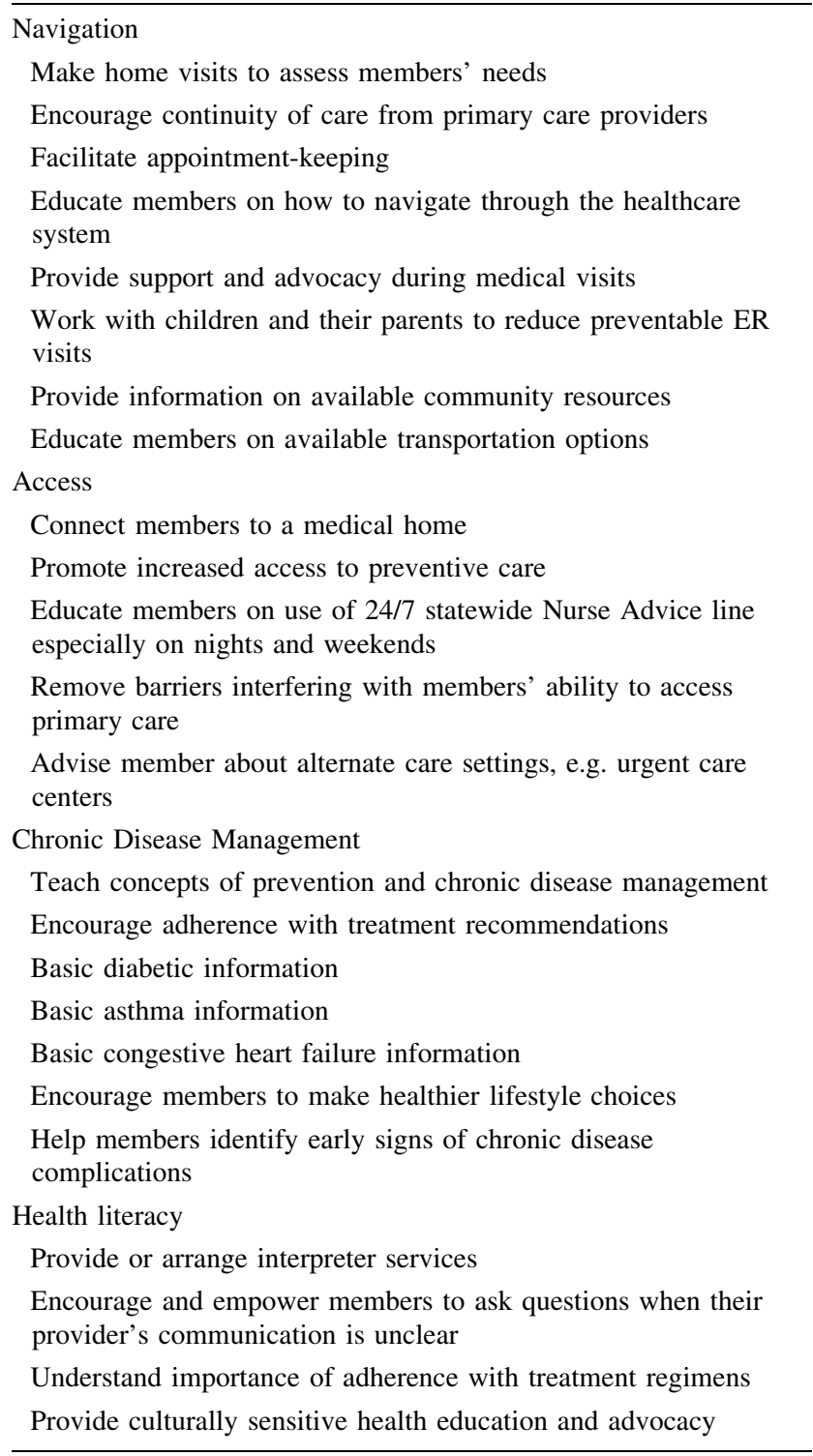

asthma, and who exhibited high use of disease management referrals, family or provider referrals, and high use of care coordination referrals. MHNM used predictive modeling using a proprietary data analysis program.

A multi-disciplinary team was developed and initially included staff from MHNM and UNM. When the program was expanded, staff from Hidalgo Medical Services (HMS), a federally qualified health center based in Silver City, New Mexico with strong links to UNM DFCM was added. The team was comprised of the MHNM medical director, health services director (an $\mathrm{RN}$ ) and a care coordinator, one UNM DFCM Coordinator, and CHWs in three sites in New Mexico-three employed by UNM DFCM and based in Albuquerque, two employed by UNM
Table 2 Field case management training subjects

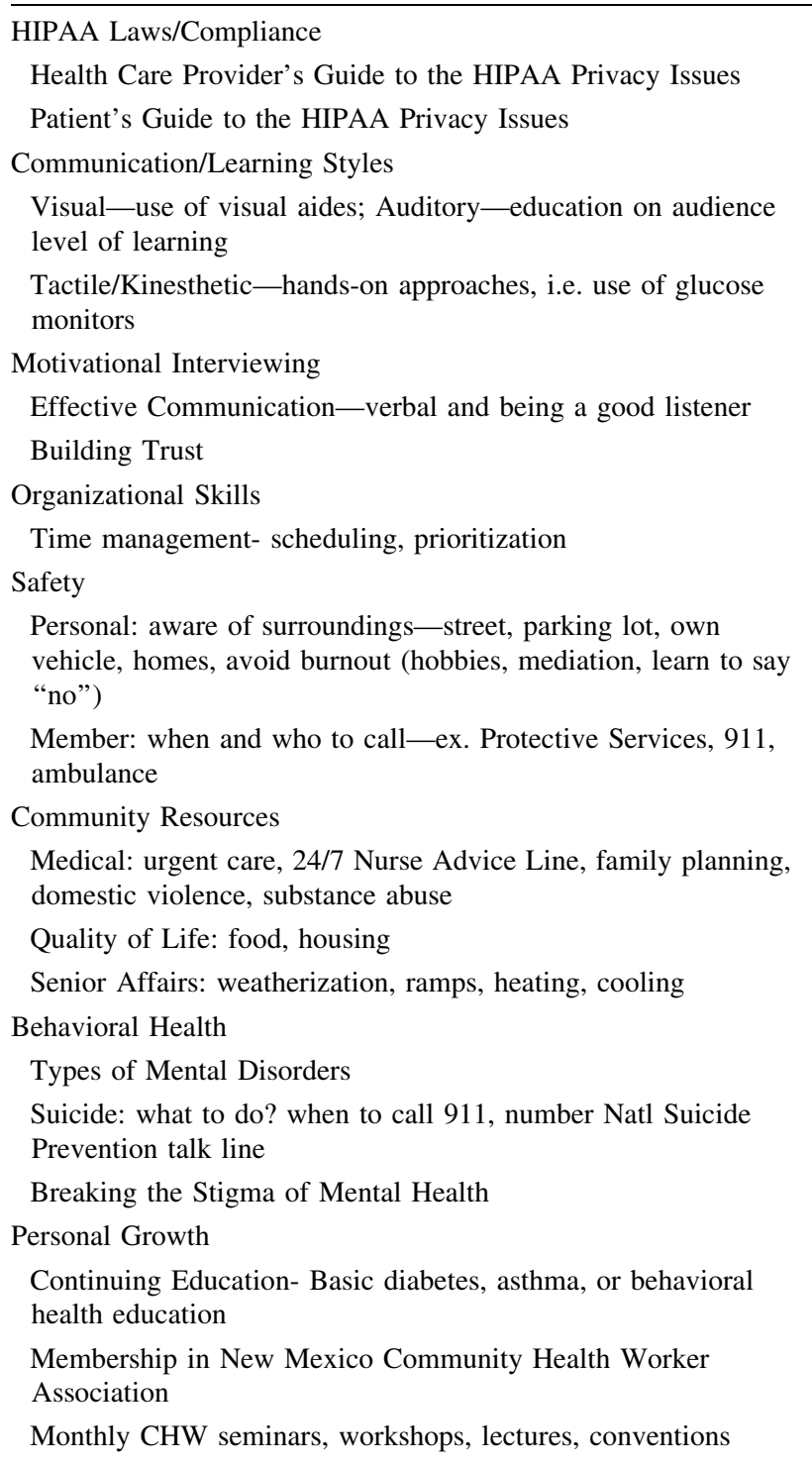

DFCM and based in Las Cruces, and one employed by HMS and based in Silver City.

CHWs underwent an initial 1 week course which covered eight topics appearing in Table 2, followed by participation in periodic in-service training sessions. WK Kellogg Foundation funding was used to support the initial startup until contract revenues began to support the program. Community resources employed to assist clients were varied and a sampling appears in Table 3.

For the purpose of this longitudinal study, we focused on the 25 month period from October 1, 2007 through October 31, 2009. During that period, there were 691 members in the program. Members were selected for the program on the basis of MHNM Reports identifying high (three or more) ED visits in a quarter. Specific characteristics associated with frequent ED use included chronic pain 
Table 3 Sample of community resources employed by client support assistants for their patients

Food bank/Utilities/Water (e.g. Salvation Army, Commodities NM, St. Vincent de Paul)

Housing Adaptation (e.g. winterization, refrigeration required for food and medicines)

Healthcare appointments (e.g. primary care, dental, counseling)

Disease management modules (e.g. diabetes, heart disease, asthma)

Pharmacy $\$ 4$ discount (e.g. Walmart, Walgreens, Target)

Durable medical equipment

SSI application assistance

Support groups (e.g. weight control, substance abuse, senior affairs)

Transportation (e.g. bus, Saferide, Sun Van)

Income Support Division

Collaboration with the NM Dept of Children, Youth and Families, and Public Schools

conditions such as back pain, abdominal pain, migraine headaches and pain from pyelonephritis. Many also had a high use of narcotics. Analysis of the narcotic use revealed that these members were obtaining narcotics from various providers simultaneously and using multiple pharmacies. Children who had frequent ED visits resulted from parents who had minimal knowledge of how to navigate the medical system. Approximately one-third of the members primarily had a diagnosis of diabetes, with a sampling of members who presented to the ED with asthma exacerbations. Members with diabetes were selected because of missed services that were easily traced-HbA1C, LDL and retinal eye examinations.

MHNM provides missed services information to the $\mathrm{CHW}$, i.e. if the member has not had the HbA1C monitoring, LDL testing, or retinal eye exam completed in the appointed time, the member is provided education on the importance of obtaining these screenings. Appointments are scheduled with the provider to ensure the missed services are completed. CHWs coordinate with the providers for completion of the missed services.

Complete, retrospective data was available for 448 members $(64 \%)$ of the total which became the study sample. Members without complete data during the time period of the study and those whose eligibility terminated either during the $\mathrm{CHW}$ intervention or after were eliminated so that the final analysis would be based on members with complete data only. The study focused on six measures: (1) Emergency Department utilization and payment, (2) Inpatient utilization and payment, (3) prescription counts and payment and (4) narcotic counts and payments, (5) PCP visits and payment, and (6) Specialist (non-PCP) visits and payment. Data on these six measures was obtained for each member for three specific timeframes as defined below. Total claims and amount paid are provided in Table 4 on the measures listed above and estimated cost savings to MHNM was then calculated.

Each time period was 6 months. The intervals for each member was determined based on the date that the member was enrolled in the program.

- Before: Six months prior to member enrollment to the program.

- During: Six months starting with the date of entry into the program. Members received $\mathrm{CHW}$ services during this time period.

- After: Seven to twelve months after the date of entry into the program. Members did not receive $\mathrm{CHW}$ services during this time period.

To estimate the magnitude of decreased resource utilization due to "regression to the mean," retrospective analysis was conducted of utilization data in another high utilizer member group in MHNM over the same period of time. This comparison group was selected on the basis of the following criteria:

1. Three or more Emergency Department visits during a 6-month timeframe from $10 / 1 / 2007$ to $3 / 1 / 2008$

2. Not enrolled in $\mathrm{CHW}$ program

3. Not Case Managed and not under Care Coordination

4. Top 448 resource consumers (not in the CHW study)

Comparable data was collected on the same six measures that were collected for the $\mathrm{CHW}$ intervention group and this data also is included in Table 4. A graphic portrayal of the differences between $\mathrm{CHW}$ intervention and non-intervention on all six measures appears in Fig. 1.

\section{Results}

Resource utilization of the 448 members who received CHW intervention and on the 448 members who did not receive intervention appears in Table 4.

Since the distributions are highly skewed, medians and ranges as well as means and standard deviations are reported. For each category, all pair-wise differences between time periods were tested using the Wilcoxon signed rank test with the Bonferroni correction for $P<0.05$.

For all measures there was a significant reduction in both numbers of claims and payments after the $\mathrm{CHW}$ intervention compared to claims and payments before or during the intervention period. There was also a significant reduction in the number of emergency department visits and payments as well as in the number of inpatient admissions during the intervention period compared to before the intervention period. 
Table 4 Resource utilization of the 448 enrollees with CHW intervention and 448 enrollees in the comparison group without intervention

\begin{tabular}{|c|c|c|c|c|c|c|}
\hline \multirow{3}{*}{$\begin{array}{l}\text { Variable } \\
\text { Emergency department }\end{array}$} & \multicolumn{6}{|c|}{6 month time period } \\
\hline & \multicolumn{2}{|l|}{ Before } & \multicolumn{2}{|l|}{ During } & \multicolumn{2}{|l|}{ After } \\
\hline & ED count & ED paid & ED count & ED paid & ED count & ED paid \\
\hline CHW & 2,655 & $\$ 650,875$ & 1,734 & $\$ 589,060$ & 815 & $\$ 225,324$ \\
\hline Non-CHW & 2,004 & $\$ 470,905$ & 612 & $\$ 182,711$ & 434 & $\$ 121,858$ \\
\hline \multicolumn{7}{|l|}{ Encounters per person $^{\mathrm{a}}$} \\
\hline \multicolumn{7}{|l|}{ CHW } \\
\hline Mean (SD) & \multicolumn{2}{|l|}{$5.9(10.6)$} & \multicolumn{2}{|l|}{$3.9(7.1)$} & \multicolumn{2}{|l|}{$1.8(4.2)$} \\
\hline Median (Range) & \multicolumn{2}{|l|}{$2(0-106)$} & \multicolumn{2}{|l|}{$1(0-46)$} & \multicolumn{2}{|l|}{$0(0-45)$} \\
\hline \multicolumn{7}{|l|}{ Non-CHW } \\
\hline Mean (SD) & \multicolumn{2}{|l|}{$4.5(1.5)$} & \multicolumn{2}{|l|}{$1.4(1.9)$} & \multicolumn{2}{|l|}{$1.0(1.5)$} \\
\hline Median (Range) & \multicolumn{2}{|l|}{$4(3-15)$} & \multicolumn{2}{|l|}{$1(0-13)$} & \multicolumn{2}{|l|}{$0(0-9)$} \\
\hline \multicolumn{7}{|l|}{ Payment per person $^{\mathrm{a}}$} \\
\hline \multicolumn{7}{|l|}{ CHW } \\
\hline Mean (SD) & $\$ 1,453(\$ 3$, & & $\$ 1,315(\$ 2$, & & $\$ 570(\$ 1, ?$ & \\
\hline Median (Range) & $\$ 355(\$ 0-\$$ & & $\$ 196(\$ 0-\$$ & & $\$ 0(\$ 0-\$ 1$ & \\
\hline Non-CHW & & & & & & \\
\hline Mean (SD) & $\$ 1,051(\$ 87$ & & $\$ 408(\$ 897$ & & $\$ 272(\$ 53$ & \\
\hline Median (Range) & $\$ 838(\$ 0-\$$ & & $\$ 107(\$ 0 \$$ & & $\$ 0(\$ 0-\$ 4$ & \\
\hline Inpatient & IP count & IP paid & IP Count & IP Paid & IP Count & IP Paid \\
\hline $\mathrm{CHW}$ & 171 & $\$ 1,056,506$ & 100 & $\$ 1,577,562$ & 29 & $\$ 183,812$ \\
\hline Non-CHW & 64 & $\$ 530,268$ & 34 & $\$ 173,880$ & 30 & $\$ 205,144$ \\
\hline Admissions per person ${ }^{\mathrm{b}}$ & & & & & & \\
\hline $\mathrm{CHW}$ & & & & & & \\
\hline Mean (SD) & $0.4(1.0)$ & & $0.2(0.8)$ & & $0.1(0.3)$ & \\
\hline Median (Range) & $0(0-13)$ & & $0(0-10)$ & & $0(0-2)$ & \\
\hline Non-CHW & & & & & & \\
\hline Mean (SD) & $0.1(0.6)$ & & $0.1(0.4)$ & & $0.1(0.5)$ & \\
\hline Median (Range) & $0(0-10)$ & & $0(0-2)$ & & $0(0-9)$ & \\
\hline Payment per person ${ }^{\mathrm{b}}$ & & & & & & \\
\hline CHW & & & & & & \\
\hline Mean (SD) & $\$ 2,358(\$ 9$, & & $\$ 3,521(\$ 3$ & & $\$ 410(\$ 2,6$ & \\
\hline Median (Range) & $\$ 0(\$ 0-\$ 12$ & & $\$ 0(\$ 0-\$ 67$ & & $\$ 0(\$ 0-\$ 4$ & \\
\hline Non-CHW & & & & & & \\
\hline Mean (SD) & $\$ 1,184(\$ 8$ & & $\$ 388(\$ 3.2$ & & $\$ 458(\$ 5,6$ & \\
\hline Median (Range) & $\$ 0(\$ 0-\$ 12$ & & $\$ 0(\$ 0-\$ 62$ & & $\$ 0(\$ 0-\$ 1$ & \\
\hline Non-narcotic & & & & & & \\
\hline Prescriptions & Rx count & Rx paid & Rx count & Rx paid & Rx count & Rx paid \\
\hline $\mathrm{CHW}$ & 22,311 & $\$ 1,079,099$ & 23,519 & $\$ 1,210,499$ & 8,311 & $\$ 379,970$ \\
\hline Non-CHW & 6.378 & $\$ 192,415$ & 4,498 & $\$ 183,180$ & 4,059 & $\$ 171,602$ \\
\hline Prescriptions per person & & & & & & \\
\hline CHW & & & & & & \\
\hline Mean (SD) & $49.8(78.3)$ & & $52.5(65.5)$ & & $18.6(23.0$ & \\
\hline Median (Range) & $12(0-383)$ & & $27(0-391)$ & & $9(0-133)$ & \\
\hline Non-CHW & & & & & & \\
\hline Mean (SD) & $14.2(19.0)$ & & $10.0(19.2)$ & & $9.1(18.2)$ & \\
\hline Median (Range) & $9(0-155)$ & & $4(0-207)$ & & $3(0-171)$ & \\
\hline
\end{tabular}


Table 4 continued

\begin{tabular}{|c|c|c|c|c|c|c|}
\hline \multirow[t]{2}{*}{ Variable } & \multicolumn{6}{|c|}{6 month time period } \\
\hline & \multicolumn{2}{|l|}{ Before } & \multicolumn{2}{|l|}{ During } & \multicolumn{2}{|l|}{ After } \\
\hline \multicolumn{7}{|l|}{ Payment per person ${ }^{c}$} \\
\hline \multicolumn{7}{|l|}{ CHW } \\
\hline Mean (SD) & \multicolumn{2}{|l|}{$\$ 2,409(\$ 6,702)$} & \multicolumn{2}{|l|}{$\$ 2,702(\$ 8,783)$} & \multicolumn{2}{|l|}{$\$ 848(\$ 1,782)$} \\
\hline Median (Range) & \multicolumn{2}{|c|}{$\$ 236(\$ 0-\$ 95,766)$} & \multicolumn{2}{|c|}{$\$ 630(\$ 0-\$ 158,893)$} & \multicolumn{2}{|c|}{$\$ 161(\$ 0-\$ 20,268)$} \\
\hline \multicolumn{7}{|l|}{ Non-CHW } \\
\hline Mean (SD) & \multicolumn{2}{|l|}{$\$ 429(\$ 771)$} & \multicolumn{2}{|l|}{$\$ 409(\$ 1,067)$} & \multicolumn{2}{|l|}{$\$ 396(\$ 2,018)$} \\
\hline Median (Range) & \multicolumn{2}{|c|}{$\$ 146(\$ 0-\$ 5,709)$} & \multicolumn{2}{|l|}{$\$ 62(\$ 0-\$ 1,314)$} & \multicolumn{2}{|c|}{$\$ 41(\$ 0-\$ 39,643)$} \\
\hline Narcotic prescriptions & Rx narc count & Rx narc paid & Rx narc count & Rx narc paid & Rx narc count & Rx narc paid \\
\hline $\mathrm{CHW}$ & 2,962 & $\$ 80,738$ & 2,748 & $\$ 105,577$ & 1,044 & $\$ 33,647$ \\
\hline Non-CHW & 827 & $\$ 13,940$ & 495 & $\$ 10,766$ & $371^{\prime}$ & $\$ 9,812$ \\
\hline \multicolumn{7}{|c|}{ Prescriptions per person $^{\mathrm{d}}$} \\
\hline \multicolumn{7}{|l|}{$\mathrm{CHW}$} \\
\hline Mean (SD) & \multicolumn{2}{|l|}{$6.6(14.7)$} & \multicolumn{2}{|l|}{$6.1(12.9)$} & \multicolumn{2}{|l|}{$2.3(5.0)$} \\
\hline Median (Range) & \multicolumn{2}{|l|}{$0(0-122)$} & \multicolumn{2}{|l|}{$1(0-108)$} & \multicolumn{2}{|l|}{$0(0-38)$} \\
\hline \multicolumn{7}{|l|}{ Non-CHW } \\
\hline Mean (SD) & \multicolumn{2}{|l|}{$1.8(3.8)$} & \multicolumn{2}{|l|}{$1.1(2.8)$} & \multicolumn{2}{|l|}{$0.8(2.2)$} \\
\hline Median (Range) & \multicolumn{2}{|l|}{$0(0-24)$} & $0(0-22)$ & & $0(0-16)$ & \\
\hline Payment per person ${ }^{\mathrm{d}}$ & & & & & & \\
\hline $\mathrm{CHW}$ & & & & & & \\
\hline Mean (SD) & $\$ 180(\$ 709)$ & & $\$ 236(\$ 1,191)$ & & $\$ 75(\$ 289)$ & \\
\hline Median (Range) & $\$ 0(\$ 0-\$ 8,096)$ & & $\$ 3(\$ 0-\$ 20,491$ & & $\$ 0(\$ 0-\$ 2,849)$ & \\
\hline Non-CHW & & & & & & \\
\hline Mean (SD) & $\$ 31(\$ 125)$ & & $\$ 24(\$ 106)$ & & $\$ 22(\$ 97)$ & \\
\hline Median (Range) & $\$ 0(\$ 0-\$ 1,794)$ & & $\$ 0(\$ 0-\$ 1,130)$ & & $\$ 0(\$ 0-\$ 94)$ & \\
\hline
\end{tabular}

${ }^{a}$ For the emergency department, the non-CHW group decreased significantly more between times 1 and $2(P<0.01)$; the CHW group decreased between times 2 and $3(P<0.01)$; and the non-CHW group decreased more over the entire study from time 1 to time $3(P<0.01)$

${ }^{\mathrm{b}}$ For inpatient there is no significant difference between the groups for changes between time 1 and time 2, but the CHW group decreased more from time 2 to time $3(P<0.01)$ and over the entire study from time 1 to time $3(P<0.01)$

${ }^{\mathrm{c}}$ For non-narcotic prescriptions, the non-CHW group decreased significantly more between times 1 and $2(P<0.01)$; the CHW group decreased between times 2 and $3(P<0.01)$; and the non-CHW group decreased more over the entire study from time 1 to time $3(P<0.01)$

${ }^{\mathrm{d}}$ For narcotic prescriptions, the CHW group decreased significantly more between times 1 and $2(P<0.01)$ and the non-CHW group decreased more between times 2 and $3(P<0.01)$; leading to no significant difference between the groups over the study interval from time 1 to time 3 $(P>0.05)$

The difference in cost from 6-months before to 6-months after CHW intervention for the 448 patients in the study sample was calculated. Costs were lower in all categories: Emergency Department-\$425,551, inpatient-\$872,694, non narcotics prescriptions-\$699,129, and narcotics prescription- $\$ 42,091$. The total cost differential was $\$ 2,044,465$ less post intervention compared to pre intervention.

The estimated program cost to MHNM to manage this high risk population of 448 members with UNM DFCMemployed and HMS-employed Community Health Workers over 25 months was $\$ 521,343$. This estimated program cost to MHNM included salaries and benefits of employees managing the CHW Program and costs from UNM Medical Group and Hidalgo Medical Services (in southwest New
Mexico) based on providing services to the members CHW individuals they manage per member/month.

Table 4 also reveals the differences in resource utilization between the 448 in the $\mathrm{CHW}$ intervention group and 448 in the non-CHW intervention group. The Emergency Department counts and cost reductions were similar between the two groups. There was a substantial difference in in-patient, prescription and narcotic counts and cost with a significantly larger reduction in resource utilization in each category in the $\mathrm{CHW}$ groups. Whereas office visits to PCPs and to specialists dropped by about half in the non$\mathrm{CHW}$ group, they remained relatively stable in the $\mathrm{CHW}$ group. Figure 1 illustrates in graphic form the relative trend differences between the two groups in all six measures. 

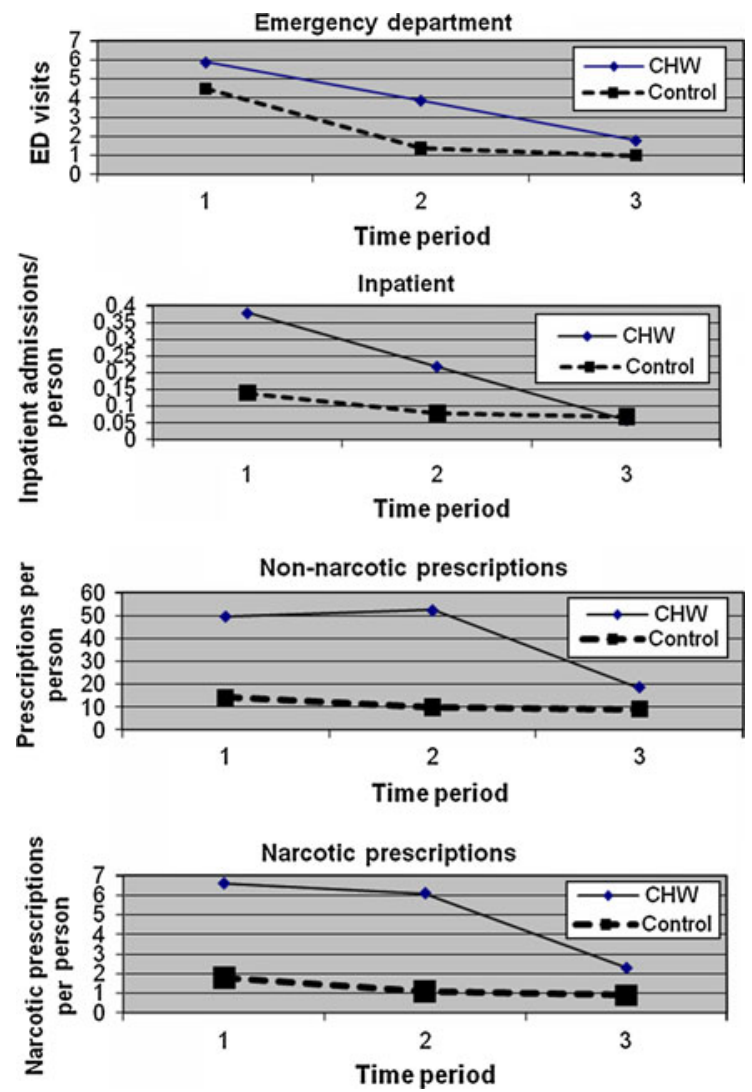

Fig. 1 Trends in resource utilization between the CHW intervention and non-CHW intervention groups

During the time that the program has been in operation, and on the basis of qualitative information, MHNM believed that the model was adding value to the healthcare of members. MHNM received positive feedback from members on the service received from CHWs and providers. They expressed appreciation for help they received in completing such preventive screenings such as HbA1C, LDL measurements, cervical cancer and breast cancer screenings. In addition, members were assisted in becoming established with medical homes and with education about alternatives to ED visits. As a result, MHNM expanded the program from the urban Albuquerque area to 13 of the state's 33 counties across the state, most of these being rural and frontier. The Program expanded further through a separate CHW contract between MHNM and Hidalgo Medical Services. The model has also attracted two other Medicaid Managed Care Organizations (MCO) in the state to contract for similar CHW Services through UNM DFCM and HMS.

\section{Discussion}

With the passage of national health care reform, by 2014 it is estimated that there will be 32 million new members enrolled in Medicaid managed care. The consequent high demand for social and community-based services that address the underlying social determinants of health and disease will require creative approaches in a constrained economic environment.

The Bureau of Labor Statistics has estimated that in 2000 there were 85,879 CHWs nationally. That number is believed to have grown $41 \%$ to 121,206 by 2005 [1]. That number is likely to increase to meet the demands for health systems to offer greater efficiency at lower cost, with increased access to government based health coverage in general and access to Medicaid in particular.

The role of CHWs described in the current study to improve access to primary care and decrease pharmaceutical and hospital based utilization was deemed a viable model and replicated by other Medicaid Managed Care Organizations in New Mexico. A broadly trained, "generalist" as opposed to a narrowly trained, disease-specific CHW appears most suitable to this managed care role. For example, with the MHNM model, CHWs are provided specific information related to the multiple ED visits, including dates of service, ED locations and, most significantly, the reason for the visit. Visits can vary from a simple sore throat, fever or headache to chronic pain, a complication of diabetes or asthma exacerbations. The CHW may assist the member along a continuum of care from establishing in a primary care medical home to helping coordinate care. Members experiencing chronic pain often reveal underlying narcotic abuse. The CHWs can offer coordinated pain management oversight. Often, members are unaware of alternatives to visiting the ED such as the availability of the 24/7 statewide Nurse Advice Line, the convenience of a nearby Urgent Care Center and, once established in a medical home, learning if that clinic has same day sick appointments for urgent needs. The authors felt this breadth of education provided to enrollees by the CHW to be vital to the success of the program.

Although there was an increase in inpatient payments from before the intervention to during the intervention, we believe that was driven by outliers wherein in a few members had more serious conditions resulting in higher inpatient costs. We believe that the number of inpatient admissions to be a more sensitive indicator of the relative success of the program in decreasing utilization.

From very large hospitals to smaller, federally qualified health centers, there is usually a hospital or clinicbased case management or social service available. However, often, those in most need of these services do not receive them. Many patients with scarce resources and complex situations, multiple diagnoses, cultural issues, and major social and economic stress avoid proactive and preventive healthcare in favor of other, more pressing priorities [13]. 
Identifying those vulnerable members of society and case identification prior to the development of serious complications will require community-based case finding, outside the walls of a clinic or hospital in settings many patients find more familiar and comfortable to access. We believe that the availability of culturally competent, easily accessed CHW services will become a key component and tool of the twenty first century healthcare system.

Kelleher and Forman coined the term, "Status One." It refers to the top one percent of users of any health system that consumes $20 \%$ of its resources and how a focus on managing this one percent brings major cost savings to that health system [14]. The Coordinated Systems of Care, a faith-based non-profit organization in Pittsburgh, implemented intense case management of their equivalent of Status One patients [15]. Their experience indicated that for case management of such high risk patients to be effective, the services had to be provided and accessed in the community rather than in the hospital, clinic or office settings. The organization actually hired staff to go into the community and find and work with these high risk patients. As in the current study, they found that in many instances the major drivers of unnecessary emergency room and other healthcare utilization were social and economic, issues amenable to field-based care coordination and case management.

Future enhancements of the program might be directed at assessing impact on other outcomes for the members who complete the CHW intervention. One possibility is to have each member complete an SF-12 Assessment (a brief, self-assessment survey of physical and mental health status) at the time of intake into the program, and have them retake the SF-12 at the time of discharge from the program [16]. Changes in aggregate SF-12 results could then be analyzed in conjunction with any changes in utilization to determine correlations.

The more challenging issue is assessing improvements in disease related outcomes. Given the relatively short duration of a given $\mathrm{CHW}$ intervention with an individual patient (3 to 6 months), it would seem unlikely that long term outcomes such as mortality and complications would be affected, furthermore, it would not be feasible to follow the members that long.

A proxy for long term outcomes might be Healthcare Effectiveness Data and Information Set (HEDIS) measures. HEDIS measures focus on process measures of healthcare that are associated with better or worse outcomes. Examples of HEDIS measures include rates of HbA1C monitoring, retinal exam, and LDL testing for members with diabetes. It should be feasible to gather available baseline information for selected HEDIS measures, perform the CHW intervention then re-measure to look for improvements.
This study was retrospective without a randomized, control design. The authors attempted to estimate the degree to which cost reductions found in the study for members with $\mathrm{CHW}$ intervention could be explained by "regression to the mean." Outcomes were measured retrospectively on the same six categories for another group of high risk, high resource-consuming members of the same size (448) but without CHW intervention identified during the same time period and followed for the same, subsequent duration. Because the two high risk groups were not from a randomized pool, the results should be interpreted cautiously. Nevertheless, differences in patterns of resource utilization over time are telling.

There was a clear regression to the mean in counts and cost in the high utilizer, non-CHW member population. However, the overall magnitude of the reduction in resource utilization appeared to be significantly higher in the CHW than in the non-CHW intervention group. Unlike the non-CHW group, the CHW group did not exhibit a significant decline in primary care and specialist resources. This can be seen as a positive outcome reflecting the impact of CHWs' assisting members in establishing with a medical home, and using appropriately primary care and specialty services for a range of needs from preventive services to chronic disease management. This emphasis on use of appropriate services may explain the significantly larger reduction in inpatient and prescription costs among CHW members than non-CHW members.

To more accurately gauge the impact on resource utilization of the CHW program, a study that is prospective and that randomizes members into and out of the $\mathrm{CHW}$ intervention is called for.

Acknowledgments We thank Patty Kehoe, RN, MPH and Ann Wehr, MD for help in creating the community health worker intervention program. This study was supported by DHHS-HRSA Health Community Access Program G920A00030 (AK) and the W.K. Kellogg Foundation- Community Voices P-0060131 and P-0105801 (WP).

Open Access This article is distributed under the terms of the Creative Commons Attribution Noncommercial License which permits any noncommercial use, distribution, and reproduction in any medium, provided the original author(s) and source are credited.

\section{References}

1. Community Health Workers National Workforce Study. (2007). United States Department of Health and Human Services, Health Resources and Services Administration, March 2007.

2. Whitle, E. M., Everhart, R. M. K., \& Wright, R. A. (2006). Measuring return on investment of outreach by community health workers. Journal of Health Care for the Poor and Underserved, $17,6-15$. 
3. Zuvekas, A., Nolan, L., Tumaylle, C., \& Griffin, L. (1999). Impact of community health workers on access, use of services, and patient knowledge and behavior. Journal of Ambulatory Care Management, 22(4), 33-44.

4. Gilliland, A. L. (2002). Beyond holding hands: the modern role of the professional doula. Journal of Obstetric, Gynecologic, and Neonatal Nursing, 31, 762-769.

5. Lacey, L., Tukes, S., Manfredi, C., \& Warnecke, R. B. (1991). Use of lay health educators for smoking cessation in a hard-toreach urban community. Journal of Community Health, 16(5), 269-282.

6. Krieger, J., Collier, C., Song, L., \& Martin, D. (1999). Linking community-based blood pressure measurement to clinical care: a randomized controlled trial of outreach and tracking by community health workers. American Journal of Public Health, 89(6), 856-861.

7. Standard Occupational Classification Action. (Modification Date Mar 12, 2009). Department of Labor Standard 2K 1094.

8. Patient Protection and Affordability Care Act. (Mar 10, 2010). Sections Appropriations (i) Definitions- Health Care Workforce (2) health professions (A) section 399v, Grants for Promotion of Health Behaviors and Outcomes, (e) Collaboration with Academic Institutions and the One Stop Delivery System, (f) Evidence Based Interventions sec. 752, Areas Health Education Centers.
9. Outcomes of community health worker interventions. Structured Abstract. Agency for Healthcare Research and Quality. Rockville, MD. http://www.ahrq.gov/clinic/tp/comhworktp.htm.

10. Murnik, M., Randal, F., Guevara, M., Skipper, B., \& Kaufman, A. (2006). Web-based primary care referral program associated with reduced emergency department utilization. Family Med, 38(3), 185-189.

11. Bissell, E., Fioranzio, C., Johnson, A., Kaufman, A., Skipper, B., \& Wagner, L. (2010). Effectiveness of a 24/7 nurse advice line on reducing non-emergent visits to the emergency room in rural New Mexico. http://hdl.handle.net/1928/10875.

12. Fiorenzio, C., \& Dulin, P. (2008). Assessment of Nurse Advice New Mexico efficacy, impact and cost/benefit (pp. 1-24). Santa Fe, NM: New Mexico Department of Health.

13. Kleinman, K. P., Gold, M., \& Makuc, D. (1981). Use of ambulatory medical care by the poor: Another look at equity. Medical Care, 19(10), 1011-1029.

14. Foreman, S., \& Kelliher, M. (1999). Status One: Breakthroughs in high risk population health management. San Francisco, CA: Jossey-Bass.

15. www.coordinatedcarenetwork.org.

16. Ware, J. E., Kosinski, M., \& Keller, S. D. (1996). A 12-item short-form health survey. Medical Care, 34(3), 220-233. 